\title{
Modelling the outskirts of galaxies in a cosmological context
}

\author{
Andrew P. Cooper ${ }^{1}$ \\ ${ }^{1}$ Institute for Computational Cosmology, Durham University \\ Science Site, South Road, Durham, DH1 3LE, UK \\ email: a.p.cooper@durham.ac.uk
}

\begin{abstract}
.
Current data broadly support trends of galaxy surface brightness profile amplitude and shape with total stellar mass predicted by state-of-the-art $\Lambda \mathrm{CDM}$ cosmological simulations, although recent results show signs of interesting discrepancies, particularly for galaxies less massive than the Milky Way. Here I discuss how perhaps the largest contribution to such discrepancies can be inferred almost directly from how well a given model agrees with the observed present-day galaxy stellar mass function.
\end{abstract}

Keywords. galaxies: evolution; galaxies: halos; galaxies: structure; methods: n-body simulations

Many remarkable observations of galaxy structure to extremely low surface brightness levels are discussed in these proceedings. These observations and quantities derived from them, such as 'stellar halo' mass, can be compared to predictions from modern cosmological simulations of galaxy formation. Such comparisons test a generic prediction that the amplitude and shape of a galaxy's surface brightness profile correlate closely with its stellar mass and even more closely with the mass of its host dark matter (DM) halo.

That prediction is founded on some of the most fundamental aspects of the theory of galaxy formation in a cold dark matter (CDM) universe. Galaxies form through the dissipative collapse of the gas trapped by the potential wells of DM halos (White \& Rees 1978; White \& Frenk 1991). Hierarchical clustering of halos leads to the accretion of additional stellar mass through mergers (see Guo \& White 2008 for a comparison of the relative contributions of merging and in situ growth to galaxies and DM halos). If the galaxy formation process was self-similar, the galactic stellar mass function would simply be a scaled-down version of the $\Lambda \mathrm{CDM}$ halo mass function. Observed luminosity functions, however, have a significantly different shape. The 'efficiency' of galaxy formation (i.e. the fraction of baryonic mass locked in long-lived stars per unit total mass) must therefore vary with halo mass. Much of the complexity of galaxy formation lies in identifying the processes (and interactions) that modulate this variation (e.g. Kauffmann, White, \& Guiderdoni 1999; Cole et al. 2000; Bower et al. 2006).

Under a simple, well motived set of assumptions, the so-called abundance matching technique can be used to estimate the trend of galaxy formation efficiency with halo mass directly from the observed galaxy stellar mass function (e.g. Guo et al. 2010). One such estimate is illustrated in the left-hand panel of Fig. 1, together with two theoretical predictions based on forward modelling. Galaxy formation efficiency peaks around the likely halo mass of the Milky Way (MW). It declines at low halo mass due to the effectiveness of supernova feedback in ejecting gas from shallow potentials, and at high masses (according to current understanding) due to the onset of AGN feedback. Recognizing that the mass ratio distributions of accreted DM halos are approximately self-similar for all host halo masses and assuming, as a simplification, that this relationship does not 

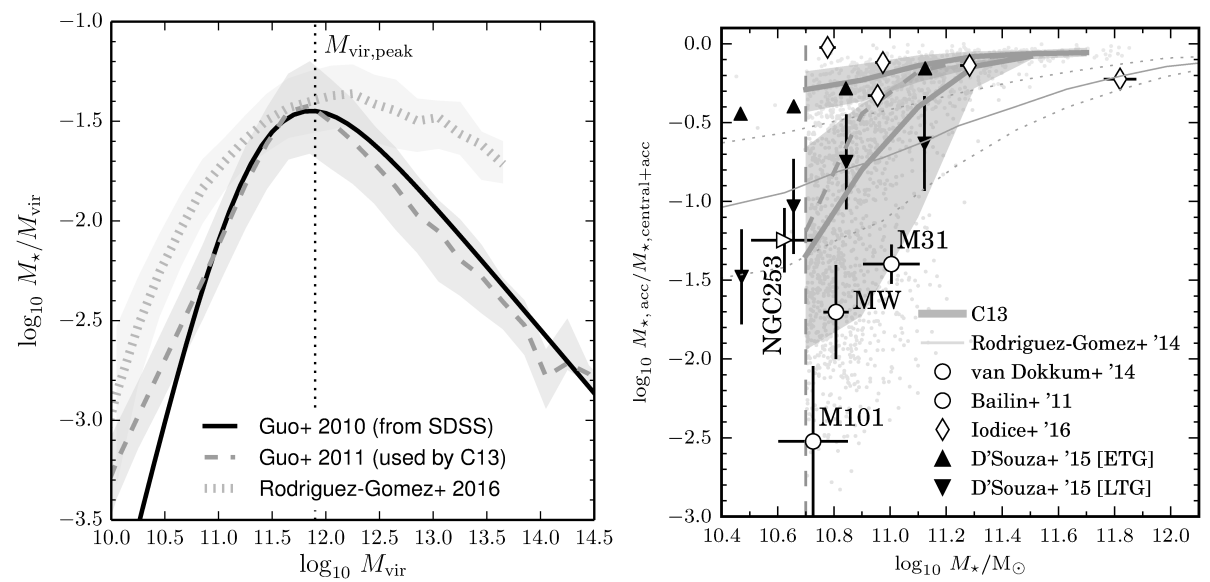

Figure 1. Left: present-day ratio of galaxy stellar mass to total mass (see text) as a function of stellar mass. The Guo et al. (2010) SDSS abundance matching result is compared to predictions of the Guo et al. (2011) model used by C13 and the Ilustris simulation used by Rodriguez-Gomez et al. (2016) (both use the SDSS mass function as a constraint). Note the log scale. Right: Mass fraction in the accreted stellar halos of galaxies vs. their stellar mass. Shaded bands bracket the $16-84^{t h}$ percentile range from C13 for early (upper) and late types (lower; split at $B / T=0.7$ ); solid lines are medians. The thick dashed line is the median of all galaxies in C13 and the vertical dashed line the mass limit of their sample. Dotted lines bracket the same range of the predictions from Illustris. Symbols show observational results (see text). Open symbols are individual galaxies, noted in legend. Filled triangles are SDSS stacks of d'Souza et al. (2014) split into early and late types by light profile concentration $C=R_{90} / R_{50}$.

evolve over time, some straightforward inferences about how the mass (fraction) of stars accreted by galaxies varies with their halo mass then follow.

Most satellites accreted by a host halo with mass $M_{\text {host }} \leqslant M_{\text {vir,peak }}$ (see Fig. 1) will have mass-to-light ratios one or more orders of magnitude higher than the host itself. The stellar halos of these galaxies will be dominated by the one or two most massive (hence most recently disrupted) progenitors. The accretion times and orbits of individual progenitors are highly stochastic and the intrinsic scatter of in situ star formation efficiency may also increase at lower mass. Hence we expect a large dispersion in the mass fraction of accreted stars for $M_{\text {host }} \leqslant M_{\text {vir,peak }}$. The are other implications; a significant difference in metallicity between deeply embedded in situ stars and diffuse accreted stars should give rise to a steep (apparent) gradient in metallicity for the host halo. Conversely, since in situ star formation is suppressed at $M_{\text {host }} \geqslant M_{\text {vir,peak }}$, the most massive satellites accreted by those hosts will have roughly similar stellar masses over a wide range of halo mass. Cooper et al. (2013 [C13]; 2015 [C15]) found that typical cluster BCGs are built by $\sim 10$ equally significant contributors. Such systems are dominated throughout by their accreted spheroids, with consequently high Sersic index, small effective radius, narrow halo-to-halo scatter and homogeneous stellar populations (e.g. De Lucia \& Blaizot 2007).

Purcell, Bullock \& Zentner (2007) were the first to make explicit predictions for accreted stellar mass fractions over a wide range of halo mass based on these ideas. More recently the problem has been tackled in greater detail by forward modelling with cosmological simulations: here we consider C13 and Rodriguez-Gomez, et al. (2016 [Illustris]). The right-hand panel of Fig. 1 compares predicted stellar halo mass fractions from these two simulations against various observational results, as a function of total stellar mass (as a more readily observable albeit noisy proxy for $M_{\text {host }}$ ). I take all these results at face value as being directly comparable to to the (unambiguously defined) total accreted 
stellar mass in the simulations. However, as discussed elsewhere in this volume, the observations use a variety of techniques which are not easy to compare even with one another. Moreover there is a fraught problem of definition (discussed in C13 and C15): generally the 'accreted mass' is inferred by extrapolating fits to data from the outskirts of galaxies inwards. The implied distinctions between accreted and in situ stars in the 'inner' galaxy differ greatly from one study to the next. An explicit, objective definition of 'the stellar halo' for a particular set of observations is important to ensure a robust comparison with models. A study carefully comparing observational inferences of stellar halo mass in galaxies of different types with different techniques would be very useful.

In Fig. 1, the Illustris relation is notably shallower than that of $\mathrm{C} 13$, with similar accreted fraction around the MW mass but much higher fractions (and smaller scatter) at lower masses, and vice versa. Comparing the $M_{\star} / M_{\text {vir }}$ relations in the left-hand panel, it is clear that Illustris predicts similar star formation efficiency around $M_{\mathrm{vir}, \text { peak }}$ but associates substantially more stellar mass with both low- and high-mass halos (presumably through enhanced in situ star formation). Following the logic above, this readily explains the different predictions of the accreted stellar mass fraction. The large scatter at low $M_{\star}$ is very clear in the observations (more evidence of this is provided in other contributions). Also clear is the separation between early and late types, at least in the SDSS stacks of D'Souza et al. (2014). Curiously, the MW and M31 both appear well below the stacked averages for late types of similar mass, albeit with the $1 \sigma$ contour of C13. At the high mass end, problems of definition become increasingly important, because it is no longer possible to distinguish two major components in the light profile. Fitting to smaller perturbations in the outskirts (see e.g. Fig. 10 of C15) can lead to 'halo' mass fractions much lower than the true fraction of accreted stellar mass.

In summary, the galaxy stellar mass function is a simple but extremely important point of comparison when interpreting discrepancies between theoretical models of stellar halos, as it is when using models to interpret observational data. Taking the issues above into account, future systematic surveys of diffuse light may be able to constrain the more subtle trends in the structure of galaxy outskirts that emerge, according to cosmological simulations, from the complex co-evolution of galaxies and their DM halos.

\section{References}

Bower, R. G., et al. 2006, MNRAS, 370, 645-655

Cole, S., Lacey, C. G., Baugh, C. M., \& Frenk, C. S. 2000, MNRAS, 319, 168-204

Cooper, A. P., et al. 2013, MNRAS, 434, 3348-3367; C13

Cooper, A. P., et al. 2015, MNRAS, 451, 2703-2722

De Lucia, G. \& Blaizot, J. 2007, MNRAS, 375, 2-14

D'Souza, R., Kauffman, G., Wang, J., \& Vegetti, S. 2014, MNRAS, 443, 1433-1450

Guo, Q. \& White, S. D. M. 2008, MNRAS, 384, 2-10

Guo, Q., White, S., Li, C., \& Boylan-Kolchin, M. 2010, MNRAS, 404, 1111-1120

Guo, Q., et al. 2011, MNRAS, 413, 101-131

Iodice, E. et al. 2016, ApJ, 820, 42

Kauffmann, G., White, S. D. M., \& Guiderdoni, B. 1993, MNRAS, 264, 201

Purcell, C. W., Bullock, J. S., \& Zentner, A. R. 2007, ApJ, 666, 20-33

Rodriguez-Gomez, V., et al. 2016, MNRAS, 458, 2371-2390

Schaye, J., et al. 2015, MNRAS, 446, 521-554

van Dokkum, P. G., Abraham, R., \& Merritt, A. 2014, ApJL, 782, L24

White, S. D. M. \& Rees, M. J. 1978, MNRAS, 183, 341-358

White, S. D. M. \& Frenk, C. S. 1991, ApJ, 379, 52-79 